\title{
Hallucinogen Dependence, Continuous Use
}

National Cancer Institute

\section{Source}

National Cancer Institute. Hallucinogen Dependence, Continuous Use. NCI Thesaurus. Code C35384.

Physical and psychological dependence on hallucinogenics, which is associated with a pattern of continuous use. 